\title{
Rare-Gas Intercalation into Fullerene Interstices
}

\author{
G. H. Kwei \\ J. D. Jorgensen \\ J. E. Schirber \\ B. Morosin
}

\author{
RECEMED \\ NOY -7 1995 \\ OSTI
}

This paper was prepared for submittal to

Symposium Proceedings - Fullerence Science and Technology

The IV International Conference on Adoanced Materials

Cancun, Mexico

August 27 to Septermber 1995

\section{August 1995}

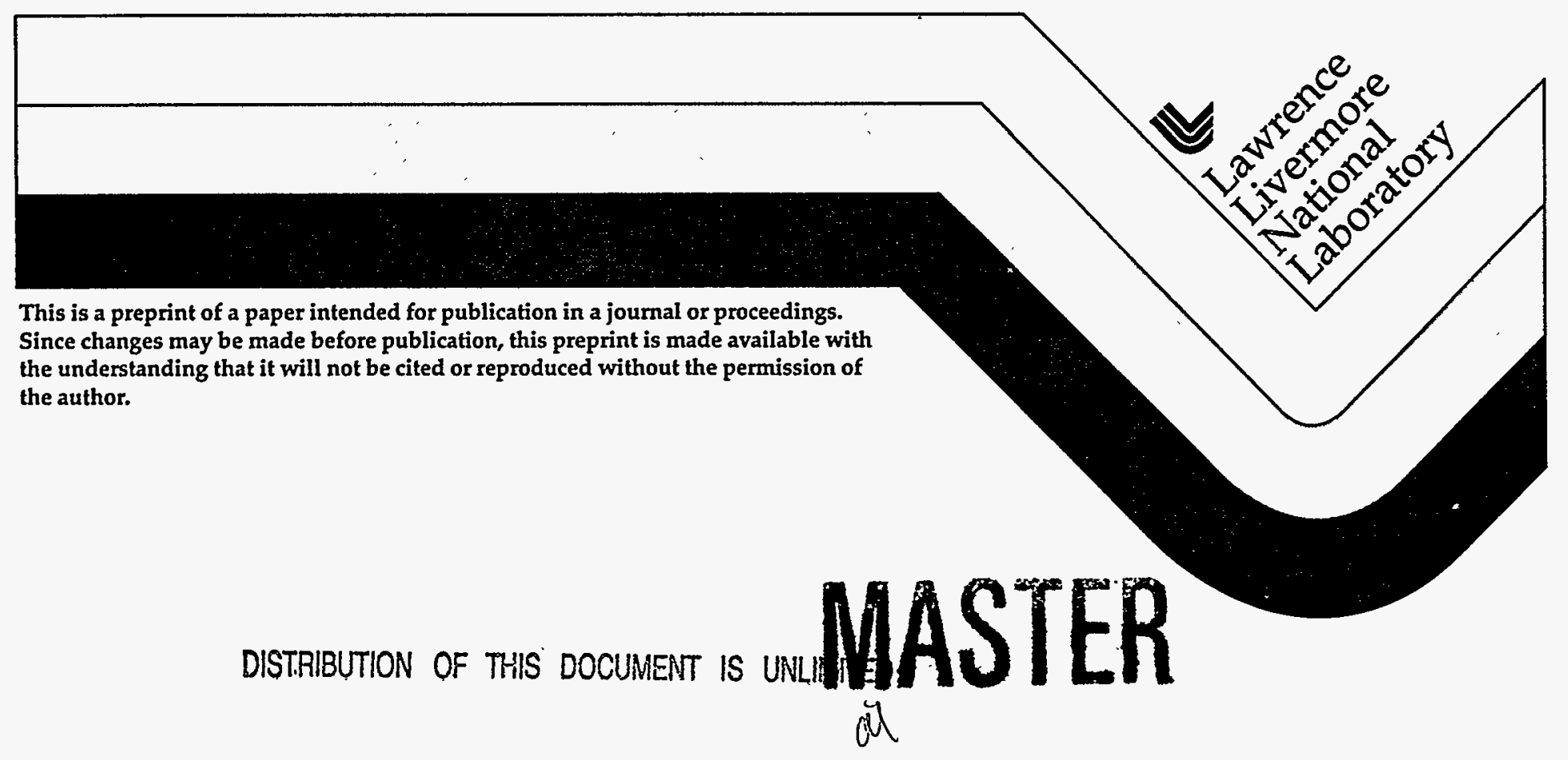




\section{DISCLAIMER}

This document was prepared as an account of work sponsored by an agency of the United States Government. Neither the United States Government nor the University of California nor any of their employees, makes any warranty, express or implied, or assumes any legal liability or responsibility for the accuracy, completeness, or usefulness of any information, apparatus, product, or process disclosed, or represents that its use would not infringe privately owned rights. Reference herein to any specific commercial product, process, or service by trade name, trademark, manufacturer, or otherwise, does not necessarily constitute or imply its endorsement, recommendation, or favoring by the United States Government or the University of California. The views and opinions of authors expressed herein do not necessarily state or reflect those of the United States Government or the University of California, and shall not be used for advertising or product endorsement purposes.

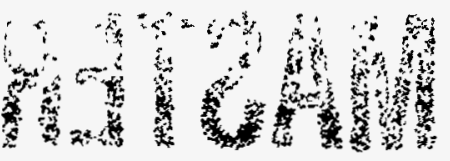


International Union of Materials Research Society (IUMRS) 1995 Meeting The IVth International Conference on Advanced Materials August 27 - September 1, 1995, Cancun, Mexico

Symposium 3: Expanded Horizon of Fullerene Science and Technology

\section{RARE-GAS INTERCALATION INTO FULLERENE INTERSTICES}

\section{George H. Kwei*, Lawrence Livermore National Laboratory, Livermore, CA} 94550

James D. Jorgensen, Materials Science Division, Argonne National Laboratory, Argonne, IL 60439

James E. Schirber and Bruno Morosin, Sandia National Laboratories, Albuquerque, NM 87185

We review our neutron diffraction studies of the intercalation of the rare gases into the interstices of $\mathrm{C}_{60}$ at pressures ranging from 0 to $6 \mathrm{kbar}$, primarily at room temperature, but also at other temperatures. Room temperature compressibilities of $\mathrm{C}_{60}$ for both the face-centered-cubic and simple-cubic phases using $\mathrm{Ar}$, which does not intercalate, as the pressure medium are in excellent agreement with the earlier work of David et al., while the values for $\mathrm{He}$ and $\mathrm{Ne}$ are slightly smaller than those using Ar. The rates of intercalation and release of $\mathrm{Ne}$, at a number of different temperatures, have been measured and show that, at a given temperature, intercalation is much slower than release. Structural refinements of the $\mathrm{Ne}$ intercalated $\mathrm{C}_{60}$ have been carried out for room temperature samples at many different pressures. These data suggest that Ne only intercalates into the octahedral hole and that the occupancy increases smoothly with pressure.

\section{Introduction}

The intercalation of atoms or molecules into porous or layered media, such as fullerenes 1,2 and zeolites ${ }^{3}$ or clays ${ }^{4}$ and graphite, 5 respectively, plays an important role in the binding or storage of atoms or molecules and has inspired a great deal of work to better understand the physics and chemistry of these processes. Intercalated materials are important technologically for their use as catalysts, waste storage media, solid-state batteries, molecular sieves, gettering materials, and in many other applications. Sometimes, intercalation endows the new material with remarkable properties. 'In the case of the fullerenes, intercalation has been a subject 
of special interest since the observation of superconductivity with alkali ion intercalants. ${ }^{6}$ There are two kinds interstitial sites in $\mathrm{C}_{60}$, one a tetrahedral site with an approximate radius of $1.1 \AA$, the other an octahedral site with an approximate radius of $2.1 \AA$; channels that connect these sites are somewhat smaller with a radius of about $0.7 \AA$. Thus the rare gases $\mathrm{He}, \mathrm{Ne}$ and $\mathrm{Ar}$, with nominal atomic radii of $0.93,1.12$ and $1.54 \AA$, respectively, should exhibit very different behavior with respect to intercalation into $\mathrm{C}_{60}$. In this article, we will review the results of our recent neutron diffraction studies of the intercalation of rare gases into fullerene interstices.

\section{Experimental Details}

$\mathrm{C}_{60}$ (99.6\% nominal purity) from commercial sources was subjected to slow progressive heating up to $400^{\circ} \mathrm{C}$ for several days in order to remove all solvents and occluded $\mathrm{N}_{2}$ and $\mathrm{O}_{2}$. The powdered sample ( $3 \mathrm{~g}$ ) was loaded into a thin walled vanadium tube which was inserted into the aluminum pressure cell described in detail elsewhere. ${ }^{7}$ Most data were collected at room temperature (296K) on the $90^{\circ}$ detector banks of the Special Environment Powder Diffractometer at Argonne National Laboratory's Intense Pulsed Neutron Source (IPNS). ${ }^{8}$ In a few cases, the pressure cell was heated (to $340 \mathrm{~K}$ ) or cooled in order to study the dependence of the structure or intercalation dynamics as a function of temperature as well. Due to shielding that is an integral part of the pressure cell, the $90^{\circ}$ scattering angle provides data that are completely free of background scattering from the walls of the cell. The maximum working pressure of this cell is $6 \mathrm{kbar}$. Typical data collection times were about one hour; however, times of about 10-20 min were used to track the initial rapid growth or decay of the lattice parameters for the time-dependent measurements and much longer times (as long as $17 \mathrm{hrs}$ ) were used in the structural refinements 
(usually from summed data from the time-dependent experiments, after the lattice parameters stopped changing). Both the structure refinement code GSAS (Generalized Structure Analysis System) ${ }^{9}$ and the in-house IPNS $\operatorname{codes}^{10}$ were used: the latter proved more amenable to the necessary modifications that allow smaller d-spacing data to be used for the more detailed structural refinements for interstitial site occupancies.

\section{Effects of Rare-Gas Atom Intercalation on Compressibility}

With $\mathrm{He}$ as the pressure medium, we know from previous attempts to measure accumulations in the lattice, that the time constant for diffusion into the lattice is very short, probably minutes. Measurements of proton NMR on $\mathrm{C}_{60}$ loaded to $1 \mathrm{kbar} \mathrm{H}_{2}$ showed that $\mathrm{H}_{2}$ is released (to half of its initial value) in $<30$ min. ${ }^{11}$ Therefore, we expected no He retention. With $\mathrm{Ne}$ and $\mathrm{Ar}$ the degree of penetration was not known, so loading as a function of time was an issue which will be described in the next Section.

The dependence of the cubic lattice parameters for $\mathrm{C}_{60}$ using $\mathrm{He}, \mathrm{Ne}$ and $\mathrm{Ar}$ rare gases as pressure media has been reported recently by us (Figure 1). la Briefly, for $\mathrm{He}$ and $\mathrm{Ne}$, the compressibilities are reduced by intercalation of the rare gas into 'the $\mathrm{C}_{60}$ lattice. These results were consistent with earlier literature studies ${ }^{12}$ in that He was found to intercalate easily, resulting in a substantially higher transition pressure for the Fm3m(face-centered-cubic or fcc) - Pa3(simple-cubic or sc) orientational-ordering pressure, while Ar did not penetrate the lattice, yielding a transition pressure typical for other nonpenetrating media such as pentane or $\mathrm{N}_{2}$. Ne was shown to slowly penetrate the lattice and the transition pressure was intermediate to that found with $\mathrm{He}$ and Ar. The lattice parameters were consistent with the change in the transition temperature in that the values were slightly larger 
for $\mathrm{He}$, and smaller for $\mathrm{Ar}$, than those for $\mathrm{Ne}$ at a particular pressure. The transition takes place with all three gases at a lattice parameter of $14.065 \AA$ with increasing pressure at room temperature compared with the value of $14.150 \AA$ of David et al. ${ }^{2}$ measured on cooling at 1 bar.

At this point, we cannot explain the slight difference in the dependence of lattice parameter on pressure between $\mathrm{He}$ and Ne. Size considerations and the refinement of the Ne site occupancies (see Section V) rule out occupancy of the tetrahedral site by Ne. However, He may be small enough to occupy the tetrahedral site. Thus the difference in behavior between $\mathrm{He}$ and $\mathrm{Ne}$ may be the additional occupancy of the tetrahedral site by He. On the other hand, the difference may be accounted for by a greater He site occupancy in the octahedral site. In either event, He occupancies are difficult to determine because of the smaller scattering length for He and because the thermal parameters, already a difficult problem in the case of $\mathrm{Ne}$, becomes even more difficult for the much lighter He.

There have been a number of determinations of the compressibility or bulk modulus of $\mathrm{C}_{60}$ using various techniques. ${ }^{13-16}$ The agreement was far from satisfactory. The first determinations ${ }^{13-14}$ involved lattice parameter measurements at high pressures $(>10-20 \mathrm{kbar})$ in diamond anvil cells. The most comprehensive measurements reported to date were those of Lundin and Sundqvist 15,16 who used a piston-and-cylinder technique to $10 \mathrm{kbar}$ on $\mathrm{C}_{60}$ at various temperatures. Unfortunately, the comparison with extrapolations of the earlier measurements ${ }^{13,14}$ differ by a factor of 2 or more. In Figure 2, we compare the bulk moduli (B = $-\mathrm{VdP} / \mathrm{dV}$ ), obtained by taking differences between adjacent lattice parameter points for data in Figure 1 and using mean values of $V$, with smoothed data from Lundin and Sundqvist. Several features are noteworthy in this figure: (1) the plots give an easily identifiable transition pressure which appears as a sharp minimum 
(considerably broader in the piston-and-cylinder work, although some of the difference in the location of their minimum from ours could arise from small temperature differences), (2) the value of the bulk modulus at $\mathrm{P}=0$ from our work (approximately $120 \mathrm{kbar}$ for all rare gases) differs substantially from the Lundin and Sundqvist value $(\approx 68 \mathrm{kbar})$, as does the pressure dependence in the fcc phase. Agreement for the sc phase is much better. (3) the bulk modulus near $6 \mathrm{kbar}$ for all gases is near $170 \mathrm{kbar}$, and is in reasonable agreement with accepted literature values.

At lower temperatures, the samples are in the simple cubic $(\mathrm{Pa} 3)$ phase and the lattice parameters depend nearly linearly on pressure. Over the pressure range 0 to $3.87 \mathrm{kbar}$ : the bulk modulus is approximately $160 \mathrm{kbar}$ at $50 \mathrm{~K}$ with $\mathrm{He}$ and 142 kbar at $100 \mathrm{~K}$ with $\mathrm{Ne}$.

\section{Absorption and Desorption Dynamics}

In our earlier work, ${ }^{1 \text { a }}$ we noticed from the measured lattice parameters that He intercalated too quickly (less than $10 \mathrm{~min}$ ) and $\mathrm{Ar}$ did not intercalate at all (in 6 days) on neutron-diffraction-experiment time scales. However, $\mathrm{Ne}$ intercalated with a time constant of hours (reaching saturation in about $24 \mathrm{hrs}$ at $2.75 \mathrm{kbar}^{1 \mathrm{~b}}$ ), making the diffusion dynamics easily accessible to TOF neutron diffraction experiments. As will be seen, further studies of the intercalation dynamics of Ne provide a fascinating story.

A large number of runs were made to study the time scales for the diffusion of Ne into the $\mathrm{C}_{60}$ lattice. ${ }^{17}$ An example is given in Figure 3, where we follow the lattice parameter changes after increasing the $\mathrm{Ne}$ pressure from $0 \mathrm{kbar}$ to $2.06 \mathrm{kbar}$ at $296 \mathrm{~K}$. The lattice parameter increases rapidly from a value of $\approx 14.081 \AA$ and 
approaches a limiting value of $\approx 14.091 \AA$ after approximately $10 \mathrm{hrs}$. The data can be fit with the expression

$$
a=a_{i}-\left(a_{i}-a_{f}\right) \exp (-t / \tau)
$$

where $a_{i}$ is the initial lattice parameter, $a_{f}$ is the final lattice parameter and $\tau$ is the time constant for diffusion. For the data in Figure $3, \tau$ has a value of $1.46 \mathrm{hrs}$. We also noticed that the measured time constants for diffusion for a given $\mathrm{C}_{60}$ sample at a given pressure would decrease on successive pressurizations to high pressure. We believe that the reason for the increasingly shorter time constants was the decrease in $\mathrm{C}_{60}$ particle size with successive pressurizations which go through the phase transition. Realizing that the measurements depended on sample history, we carried out a series of measurements (of which the data shown in Figure 3 is one) involving pressurizations to various pressures below $2.06 \mathrm{kbar}$ (e.g. below the phase transition) on a sample that had been previously pressurized to $4.12 \mathrm{kbar}$. In this series of experiments, we find that the dependence of time constant on loading pressure gives a straight line (Figure 4).

In all cases where the Ne pressure is released after the system has reached saturation, we find that the change in lattice parameter occurs too quickly to be measured. Figure 5 shows this behavior in the lattice parameter when the Ne pressure is released from $4.12 \mathrm{kbar}$ to $1.03 \mathrm{kbar}$. This asymmetric diffusion clearly shows that the activation energy for loading is much larger than that for release.

Although release occurred too quickly to be measured at room temperature, the diffusion rate becomes accessible when the temperature is lowered. For example, when $\mathrm{Ne}$ is released to a pressure of $0 \mathrm{kbar}$ from $1.51 \mathrm{kbar}$ at $200 \mathrm{~K}$, the time constant for the equilibration of the lattice parameters is $1.40 \mathrm{hrs}$ (as shown in Figure 6), almost the same time constant as that for loading in the experiment shown in Figure 3 in which the temperature was $96 \mathrm{~K}$ higher! 


\section{Pressure Dependence of Ne Site Occupancies}

A number of long runs, in addition to numerous data sets obtained from summed sequential runs (after the system had reached equilibrium) from the timedependent studies provided data at various Ne pressures with good statistics (corresponding to total data collection times of 5-17 hrs) for Rietveld structure refinement of the $\mathrm{Ne}$ interstitial site occupancies. $1 \mathrm{~b}$

In order to determine the interstitial site occupancies for $\mathrm{Ne}$, we carried out least-squares refinements of the structure using a modified version of the Rietveld code available at IPNS. The modifications included the use of spherical scattering form factors for the rotating $\mathrm{C}_{60}$ and a contribution to the background derived from the expected diffuse scattering arising from disorder. 18 These modifications allowed reasonably good refinements to be carried out, but the background was still not adequately modeled at d-spacings below about $1.3 \AA$. To extend the $d$-spacing range of the refinements to about $0.55 \AA$, we used an additional fourier-filtering background-fitting technique. ${ }^{19}$ A portion of the data, together with the fitted profile, is shown in Figure 7.

From our least-squares refinements, it was immediately clear that all of the intercalated Ne was in the octahedral sites. Tetrahedral site occupancies were found to be essentially zero at all pressures, and set to zero in all further refinements. It was found that even with the inclusion of data to d-spacings as small as $0.55 \AA$, we could not remove the correlation between site occupancies and thermal parameters and independently refine these parameters. We feel that this occurs because the $\mathrm{Ne}$ atom sits in a large irregularly shaped "box" bound by six $\mathrm{C}_{60}$ molecules, and that this "square well" potential is not adequately modeled by the harmonic potentials in the refinement codes. In order to calculate site occupancies, we fixed the thermal 
parameter for $\mathrm{Ne}$ at $\mathrm{B}=13 \AA^{2}$. This corresponds roughly to a cage in which the $\mathrm{Ne}$ atom can move relatively freely about a central position by about $0.4 \AA$ in the octahedral site and gives a site occupancy approaching unity at high pressures. If we assumed a value of $5 \AA^{2}$ for $B$, which is almost certainly too low an estimate, then the occupancies at the highest pressures decrease to $\mathrm{n}_{\mathrm{Ne}}=0.67$. The values of the Ne site occupancies at various pressures using the refinements with $B=13 \AA^{2}$ is shown in Figure 8. This shows that the equilibrium Ne site occupancy changes reversibly with changing Ne pressure until it saturates at high pressures.

\section{Conclusions}

We have reported recent results from our neutron scattering studies of the intercalation of rare gases into $\mathrm{C}_{60}$ at various pressures. These include the study of the changes in the lattice parameters with pressure and the determination of the pressure dependence of the compressibility at various temperatures, studies of the dynamics of intercalation and release at various pressures and temperatures and, finally, refinement of the structure in the face-centered cubic (Fm3m) phase to determine the pressure dependence of the Ne site occupancies in the interstices of $\mathrm{C}_{60}$. At the present time, we.are studying theoretical models that correlate the observed behavior. Much work remains to be done: we are continuing a more detailed study of the activation energies for the asymmetrical rates for diffusion into and out of $\mathrm{C}_{60}$, we are carrying out refinements of the interstitial occupancies of $\mathrm{Ne}$ for the simple cubic ( $\mathrm{Pa3}$ ) phase of $\mathrm{C}_{60}$ at higher pressures and at lower temperatures, and we plan to begin soon the study of the intercalation of other gases.

\section{Acknowledgments}


We thank R.L. Hitterman and S. Short for their considerable help in data collection and analysis. This work is supported by the U.S. Department of Energy and funded in part by its Office of Basic Energy Sciences (OBES), Division of Materials Sciences (DMS), under contract W-7405-ENG-48 at Lawrence Livermore National Laboratory, contract W-31-109-ENG-38 at Argonne National Laboratory, and contract DE-AC04-94AL85000 at Sandia National Laboratories. IPNS at Argonne National Laboratory is funded as a national user facility by the OBES/DMS. 


\section{References}

*To whom correspondence should be addressed.

1. (a) J.E. Schirber et al., Phys. Rev. B, 1995, 51, 12014; (b) B. Morosin et al., Phys. Rev. Lett. (submitted July, 1995).

2. W.I.F. David et al., Europhys. Lett., 1992, 18, 219; Proc. Roy. Soc. Lond. A, 1993, 442, 129 (1993); and J. Phys.: Condens. Matter, 1993, $\underline{5}, 7923$.

3. D.W. Breck, Zeolite Molecular Sieves, (J. Wiley, New York, 1974), pp. 379.

4. R.E. Grim, Clay Mineralogy, (McGraw-Hill, New York, 1968), pp. 471.

5. J.J. Kipling and R.B. Wilson, Trans. Faraday Soc. 1960, $\underline{56}$, 562; M.M. Dubinin, O. Kadlac and A. Zukal, Nature, 1965, 205, 75; and T.G. Lamond, J.E. Medcalf and P.L. Walker, Carbon, 1965, $\underline{3}, 59$.

6. A.F. Hebard et al., Nature (London), 1991, $\underline{350,600 .}$

7. J.D. Jorgensen et al., Physica C, 1990, 171, 93.

8. J.D. Jorgensen et al., J. Appl. Crystallogr., 1989, 22, 321.

9. A.C. Larson and R.B. Von Dreele, Los Alamos National Laboratory Report, LA-UR-86-748 (1987).

10. R.B. Von Dreele, J.D. Jorgensen and C.G. Windsor, J. Appl. Crystallogr., $1982, \underline{15}, 581$.

11. R.A. Assink et al., J. Mater. Res., 1992, I, 2136.

12. G.A. Samara et al., Phys. Rev. B, 1993, 47, 4756.

13. J.E. Fischer et al., Science, 1991, 252, 1288.

14. S.J. Duclos et al., Nature (London), 1991, 351, 380.

15. A. Lundin et al., Solid State Commun., 1992, 84, 879.

16. A. Lundin and B: Sundqvist, Europhys. Lett, 1994, 20, 463.

17. J.D. Jorgensen et al., unpublished. 
18. J.R.D. Copley et al., J. Phys. Chem. Solids, 1992, 53, 1353; J. Phys.:

Condens. Matter , 1993, $\underline{5}, 4353$. See also M. Atoji, T. Watanabe and W.N. Lipscomb, Acta Cryst., 1953, 6, 62.

19. J.R. Richardson, Jr., and J. Faber, Jr., Adv. in X-ray Analysis, 1985, 29,

143. 


\section{Figure Captions}

1. Lattice parameters vs. pressure for $\mathrm{C}_{60}$ at room temperature: $\boldsymbol{Q}, \Delta$ and $\nabla$ denote $\mathrm{He}, \mathrm{Ne}$ and Ar data, respectively. The Ne points are numbered in the order they were taken.

2. Bulk modulus $B$ (defined as $-V d P / d V$ ) vs. pressure for $\mathrm{C}_{60}$ at room temperature: $\boldsymbol{Q}, \Delta$ and $\nabla$ denote $\mathrm{He}, \mathrm{Ne}$ and $\mathrm{Ar}$ data, respectively. The dashed curve is from the data of Lundin and Sundqvist (Reference 12).

3. Lattice parameter vs. time for a $\mathrm{C}_{60}$ sample at room temperature with $\mathrm{Ne}$ pressure loaded from an initial value of $0.0 \mathrm{kbar}$ to a final value of $2.07 \mathrm{kbar}$.

4. Plot of the time constant for room temperature loading $\mathrm{Ne}$ into $\mathrm{C}_{60}$ at a number of pressures.

5. Lattice parameter vs. time for a $\mathrm{C}_{60}$ sample at room temperature with $\mathrm{Ne}$ pressure released to $0.0 \mathrm{kbar}$ from an initial value of $2.75 \mathrm{kbar}$.

6. Lattice parameter vs. time for a $\mathrm{C}_{60}$ sample at $200 \overline{\mathrm{K}}$ with Ne pressure released to $0.0 \mathrm{kbar}$ from an initial value of $1.51 \mathrm{kbar}$.

7. Neutron diffraction data for $\mathrm{C}_{60} \mathrm{Ne}_{\mathrm{x}}$ at a pressure of $1.55 \mathrm{kbar}$. The continuous line through the sets of points is the calculated profile from the Rietveld refinement, the set of tick marks below the data indicate the positions for the allowed reflections, and the lower curve represents the difference between observed and calculated profiles.

8. Dependence of the interstitial site occupancies for $\mathrm{Ne}$ in the octahedral sites in $\mathrm{C}_{60}$ on Ne pressure (from Reference $1 \mathrm{~b}$ ). The value for the Ne isotropic thermal parameter $B$ is set to $13.0 \AA^{2}$ in the structural refinements. 


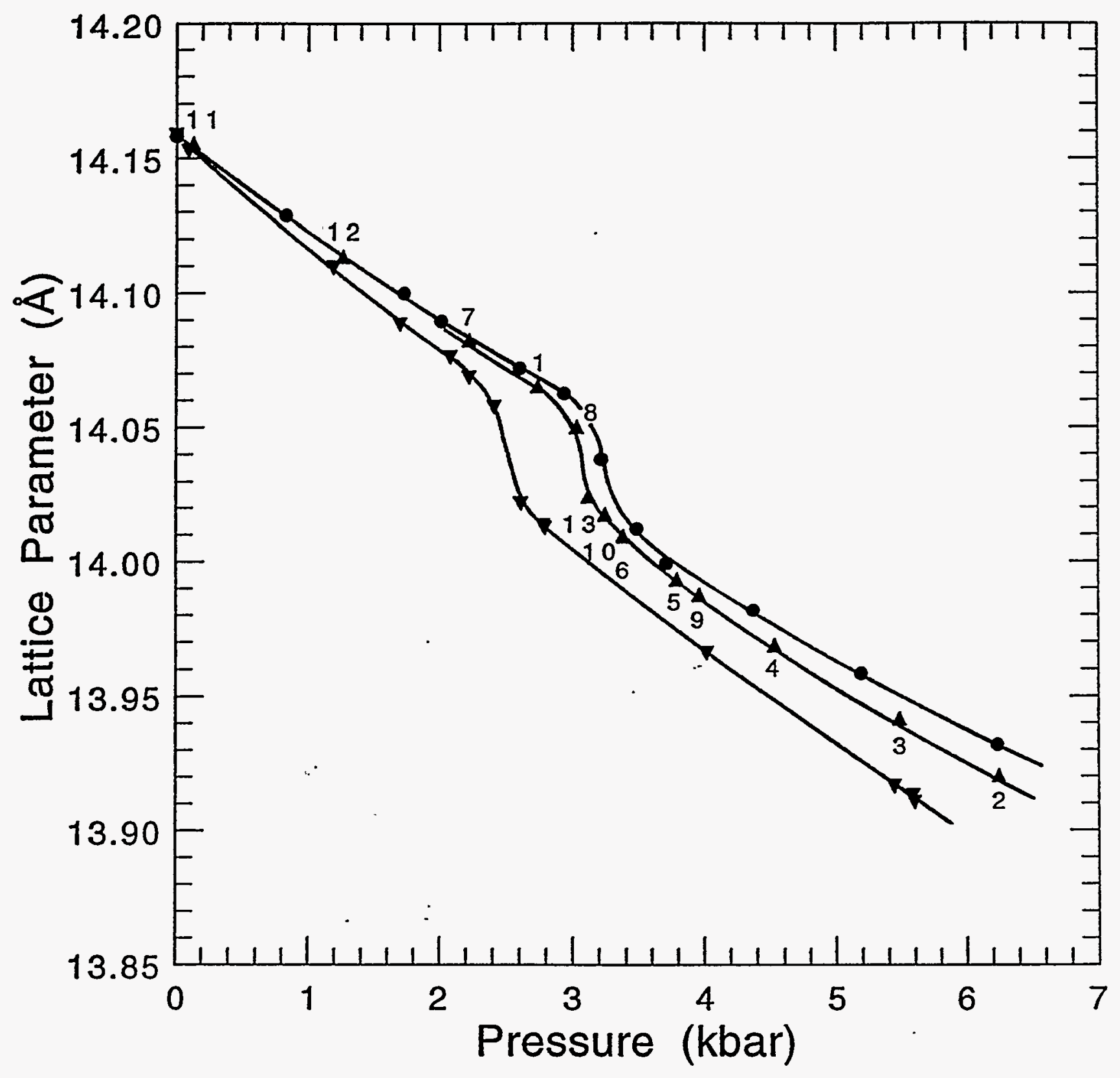




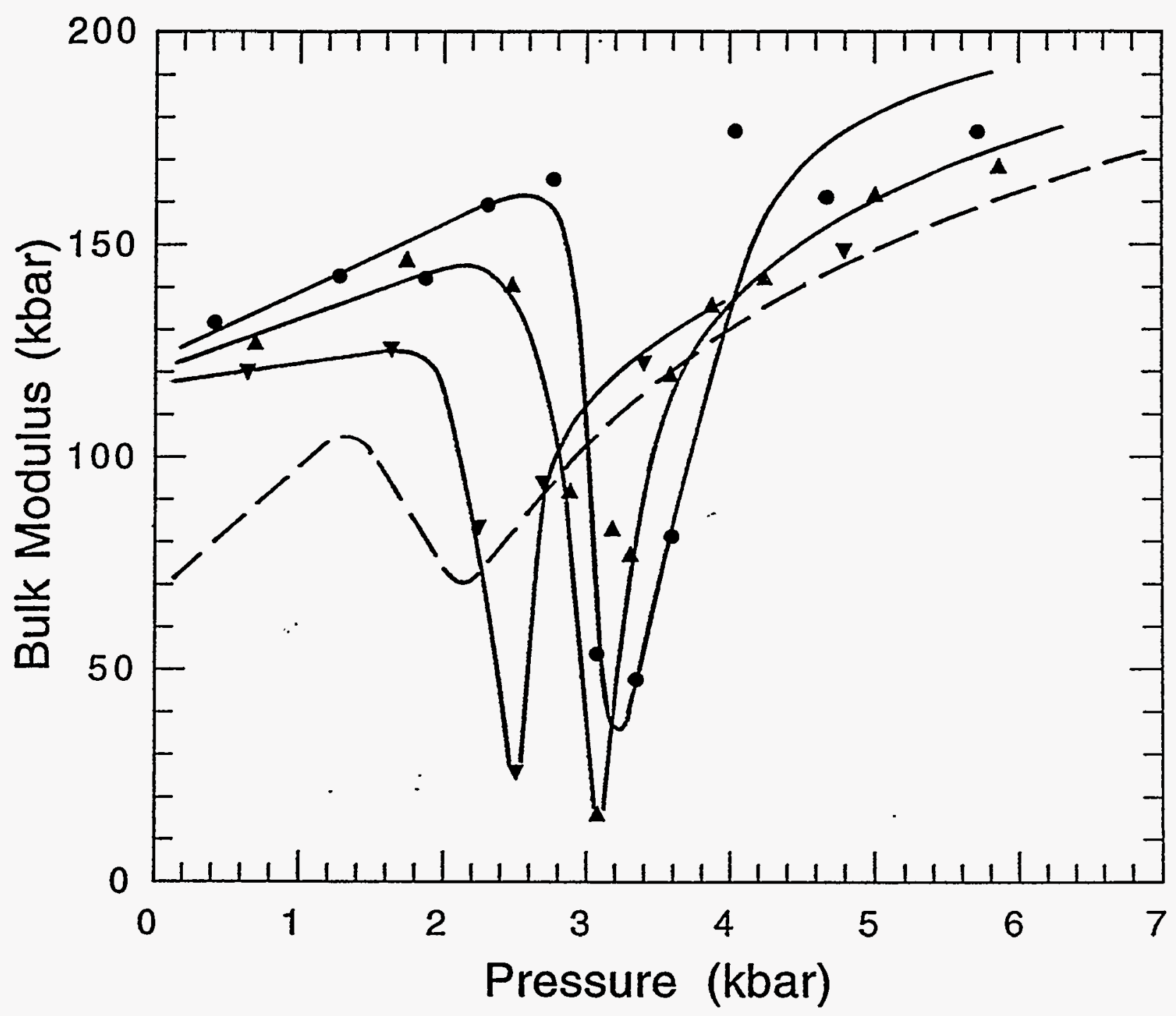




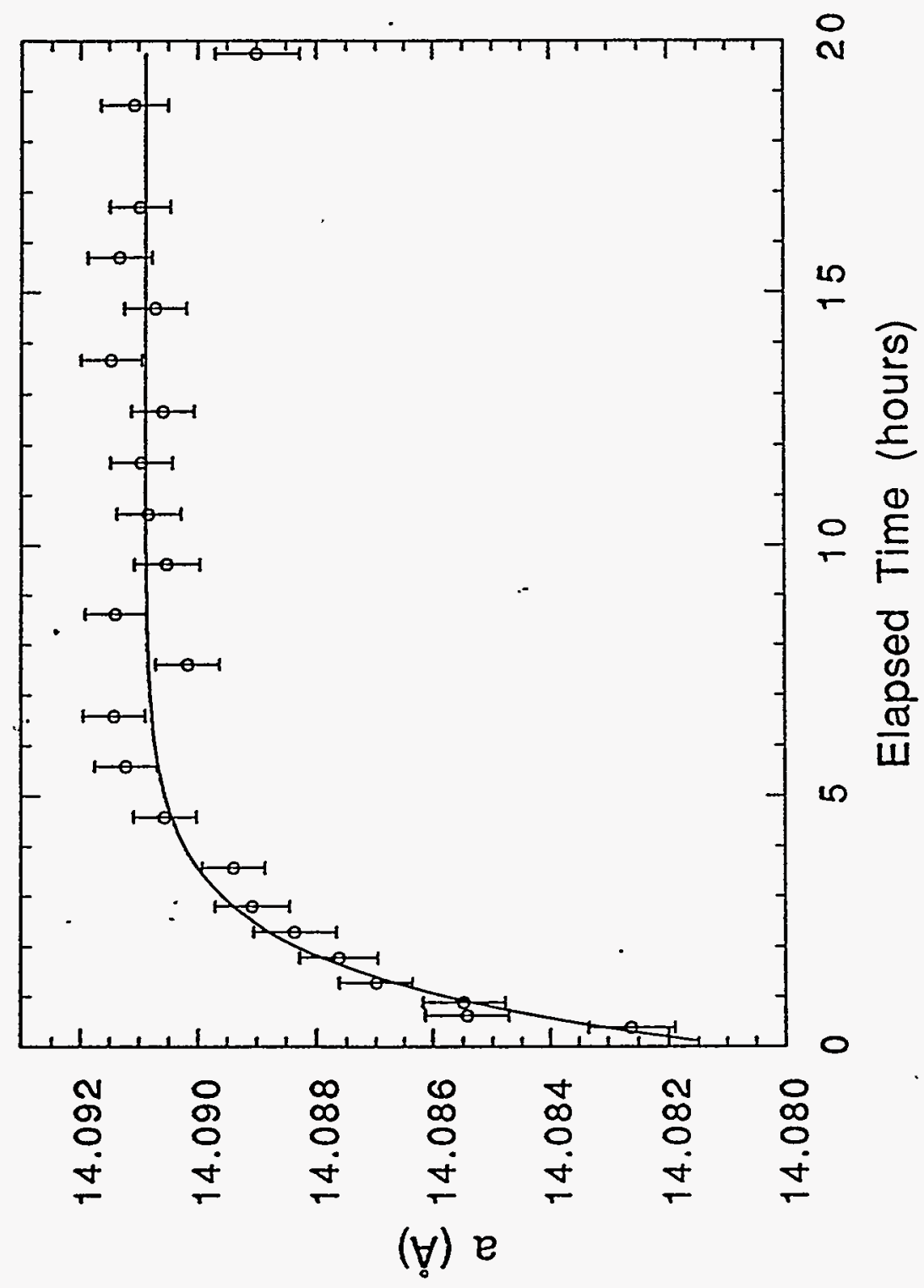




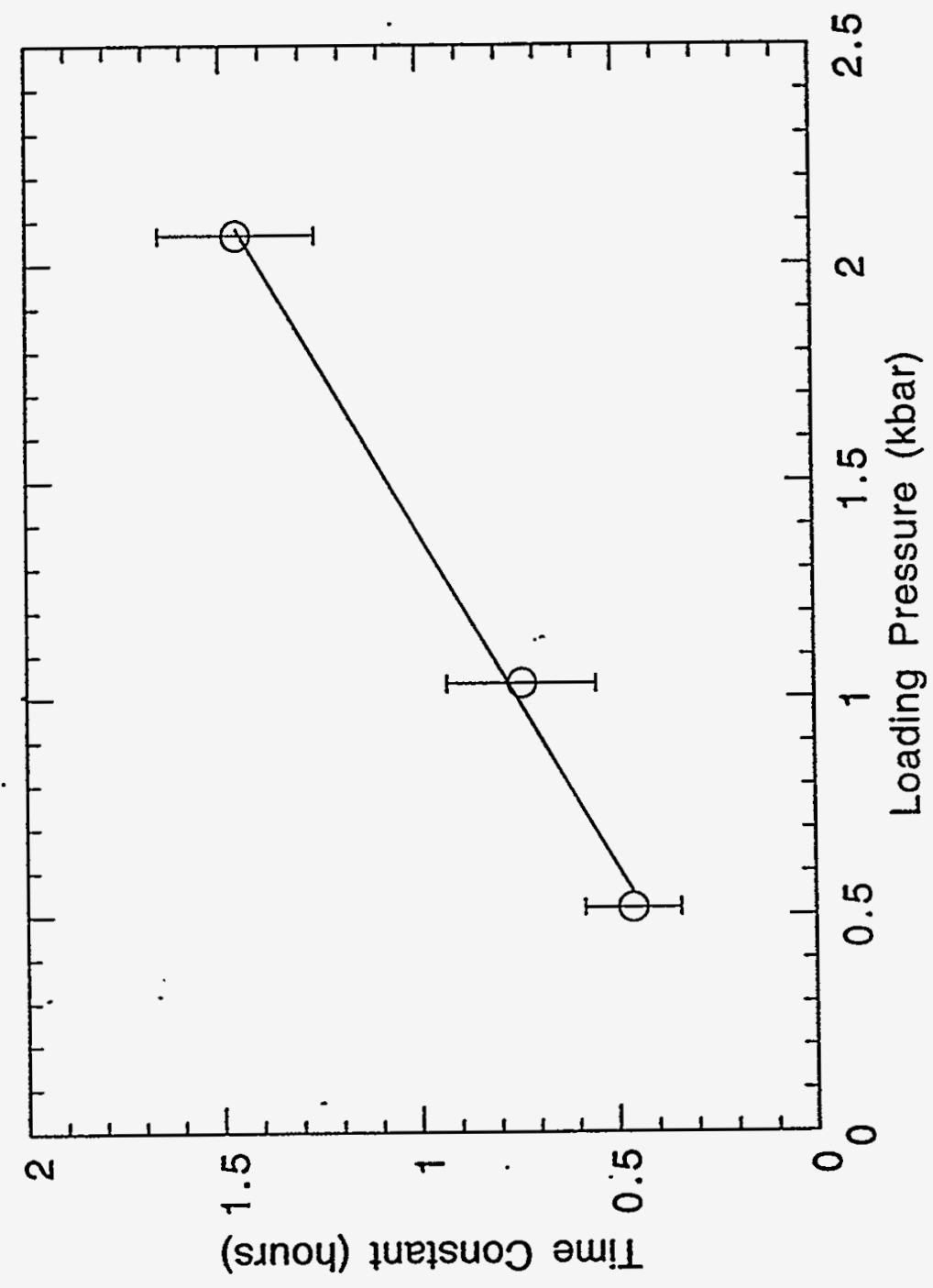




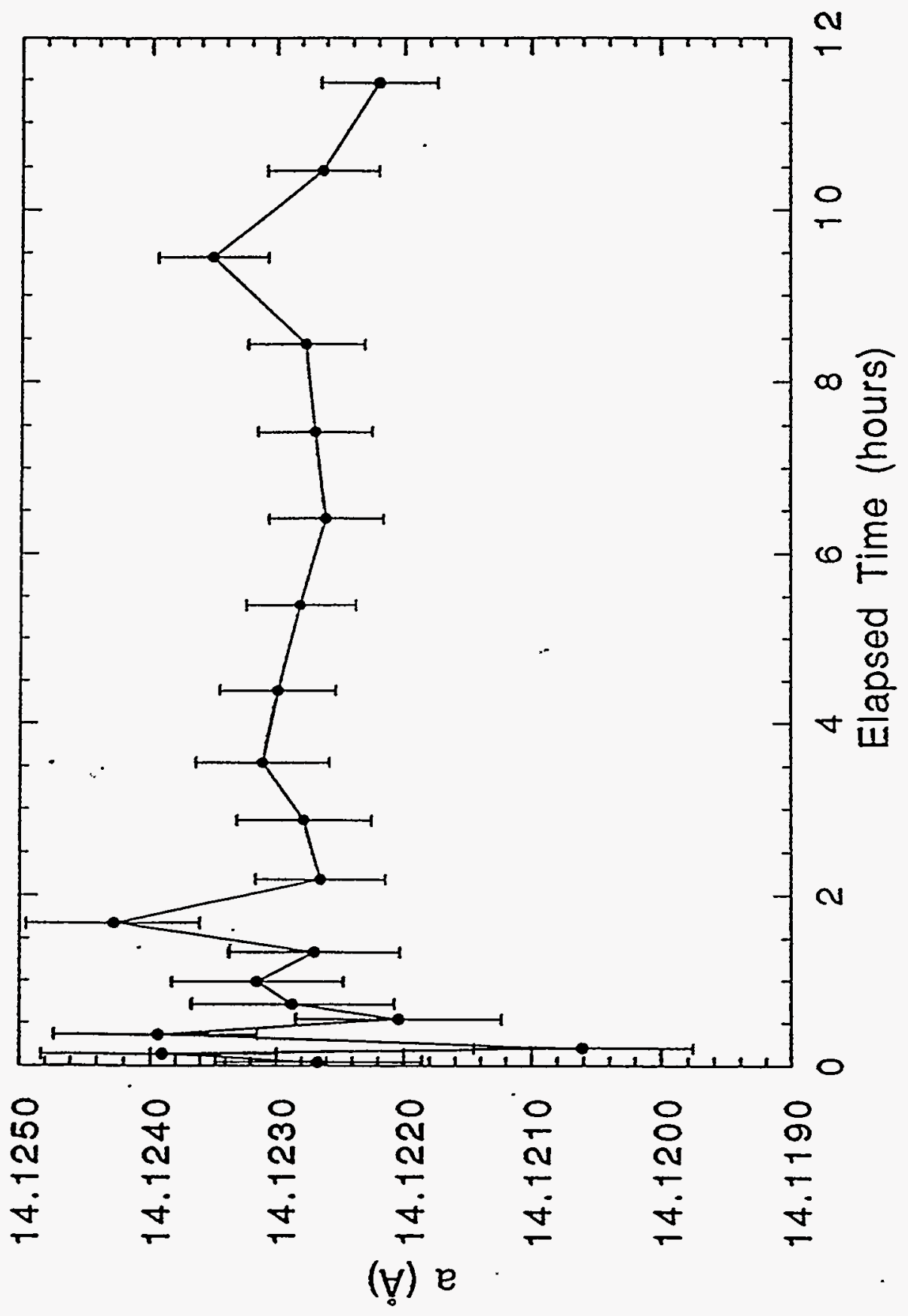




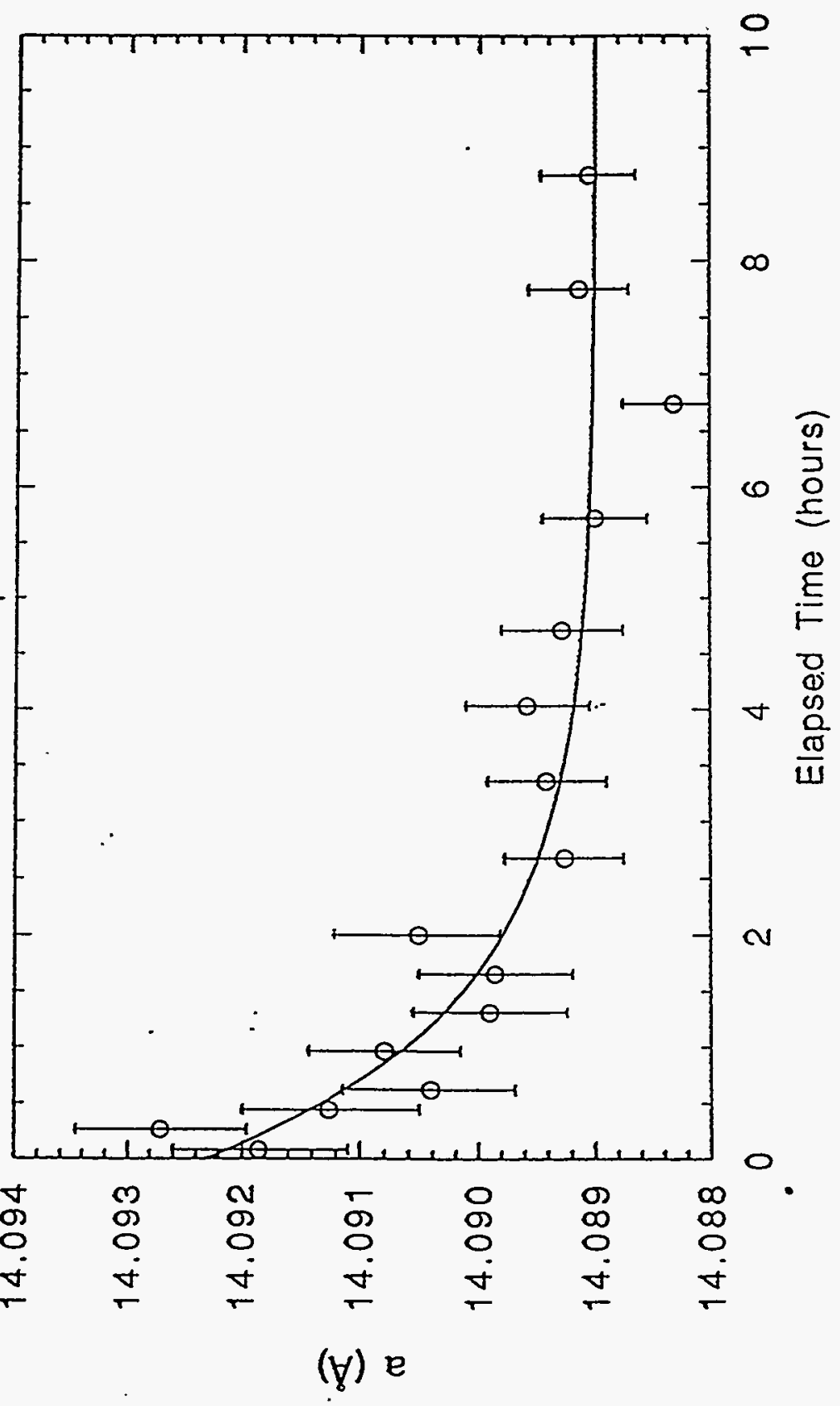




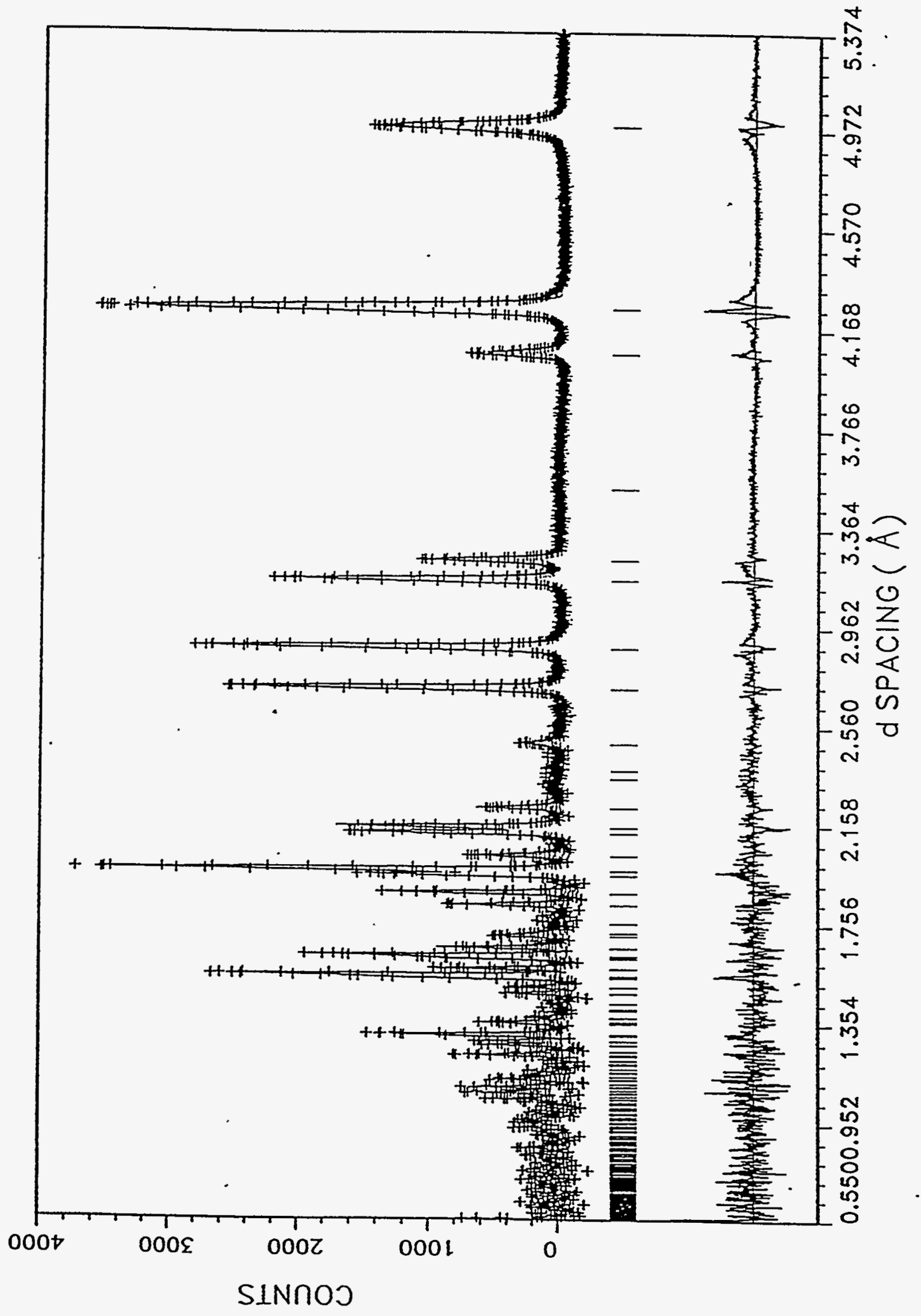




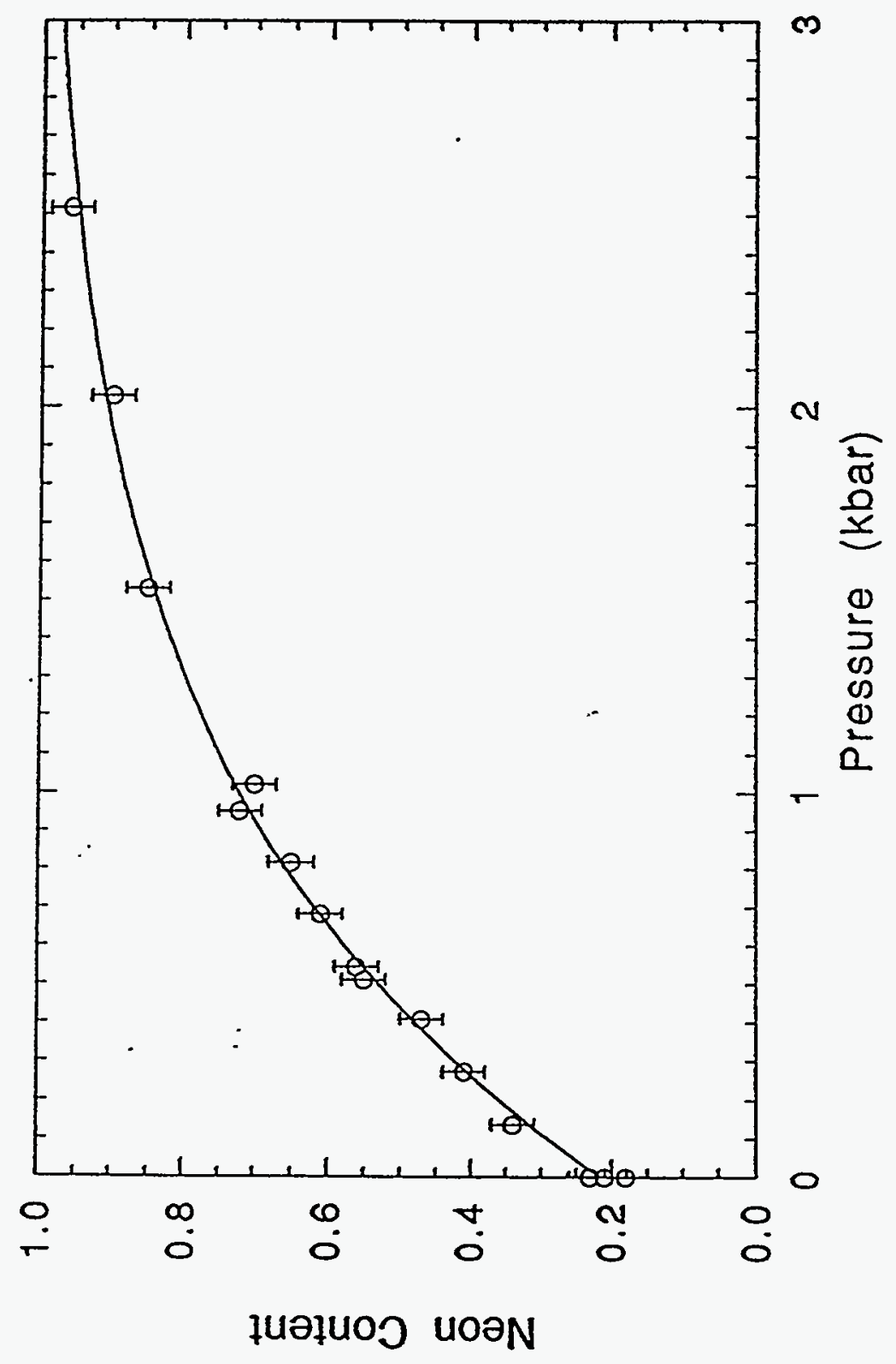


